\title{
Desarrollo de aplicación móvil para niños con dificultades de aprendizaje de la lectura y escritura
}

\author{
Carlos Máñez-Carvajal ${ }^{*}$ y Jose Francisco Cervera-Mérida \\ Universidad Católica de Valencia San Vicente Mártir, c/ Sagrado Corazón, 5. 46110 Godella-España \\ (correo-e: carlos.manez@ucv.es; josefran.cervera@ucv.es) \\ * Autor a quien debe ser dirigida la correspondencia.
}

Recibido Jul. 7, 2021; Aceptado Sep. 9, 2021; Versión final Oct. 29, 2021, Publicado Feb. 2022

\begin{abstract}
Resumen
El presente trabajo tiene como objetivo el desarrollo de una aplicación móvil para niños con dificultades específicas de aprendizaje de lectura y escritura. La aplicación incide en los procesos de conversión entre los sonidos y los grafemas de las sílabas, facilita el procesamiento lingüístico y de memoria de trabajo, y refuerza los procesos de conciencia fonológica. Se emplea Basic4android para llevar a cabo el desarrollo informático. El resultado es una aplicación que permite a los docentes decidir la complejidad de las sílabas y palabras que aparecen en los ejercicios. Consta de 225 palabras clasificadas según su longitud y complejidad silábica y cuenta con un sistema de ayudas y refuerzos que la hacen amigable para los niños. En conclusión, el software desarrollado posibilita acceder a los contenidos en cualquier momento y lugar, sin necesidad de Internet, permitiendo a los estudiantes una mayor flexibilidad para trabajar a su propio ritmo.
\end{abstract}

Palabras clave: tecnología educativa; aplicación móvil; dificultades específicas; aprendizaje; lectura; escritura

\section{Development of a mobile application for children with reading and writing learning difficulties}

\begin{abstract}
The present study develops a mobile application for children with specific learning difficulties in reading and writing. The application enhances conversion processes between sounds and graphemes, facilitates linguistic and memory processing, and reinforces phonological awareness processes. Basic4android is used to develop the app. The result is an application that allows teachers to decide syllable and word complexity for school exercises. The application contains 225 words classified according to length and syllabic complexity. It also has an in-built help and reinforcement system that makes the app friendly for children. In conclusion, the application developed here facilitates access to school content at any time and place, even without Internet, providing students with greater flexibility for studying at their own pace.
\end{abstract}




\section{INTRODUCCIÓN}

El uso de dispositivos móviles en el ámbito educativo se ha convertido en los últimos años en una realidad habitual en el proceso enseñanza aprendizaje de los alumnos, independientemente de su edad o de la asignatura que cursen. Estudiantes y profesores pueden utilizar los dispositivos inteligentes para acceder a recursos educativos, conectarse con otros o crear contenidos, tanto dentro como fuera de las aulas. El uso de los dispositivos móviles facilita a los estudiantes la posibilidad de tomar control de su propio aprendizaje, en un contexto donde el estudiante se convierte en actor central del proceso de aprendizaje (Kraut, 2013).

El tamaño reducido de los teléfonos celulares, PDA o tabletas permite a los estudiantes acceder a los contenidos en cualquier momento y lugar, posibilitando a los estudiantes una mayor flexibilidad para trabajar a su propio ritmo y aumentando su motivación para buscar nuevas oportunidades de aprendizaje. Estos dispositivos tienen un coste económico reducido en comparación con los libros o los ordenadores de sobremesa (Politi-Georgousi y Drigas, 2020) y permiten una personalización y adaptación de la presentación de los contenidos en función de las capacidades y habilidades de los usuarios individuales.

La informática posibilita la creación ilimitada de aplicaciones independientemente del sector económico o industria (Alarcón-Aldana et al., 2016; Cáceres y Ossadón, 2018; Choque-Tolmo et al., 2020), en la última década, el interés creciente las tecnologías móviles ha favorecido la creación de numerosas iniciativas de carácter innovador incrementándose de manera exponencial el número de desarrollos de aplicaciones con propósitos educativos. Un sistema operativo muy extendido en teléfonos inteligentes y tabletas es Android, basado en Linux y diseñado principalmente para el tacto de las pantallas de este tipo de dispositivos. Los desarrolladores disponen de varias herramientas para generar aplicaciones compatibles con el sistema operativo Android como por ejemplo Eclipse, Basic4android, Android Studio o App Inventor (Gironés,2018; Papadakis et al.,2017).

Según Pressman (2010) las actividades fundamentales y sus resultados en el desarrollo del software son: 1) especificación del software: los clientes e ingenieros definen el software a producir, 2) desarrollo de software: diseño y programación, 3) validación: corroboración de que el producto es lo que el cliente desea y; 4) evolución del software: modificación para adaptarlo a las necesidades de los clientes y del mercado. Entre los modelos más extendidos para desarrollar software destacan el modelo en cascada, el desarrollo incremental o la ingeniería de software orientada a la reutilización. No se excluyen mutuamente y en muchas ocasiones se utilizan de forma conjunta, sobre todo en desarrollos de sistemas grandes o complejos. El desarrollo incremental (Figura 1) se caracteriza porque permite crear versiones cada vez más completas del software. Las actividades de especificación, desarrollo y validación están entrelazadas en vez de separadas. Cada incremento o versión del software cuenta con la retroalimentación de los clientes finales, reduciendo el riesgo de elaborar una solución informática que no satisfaga sus necesidades.

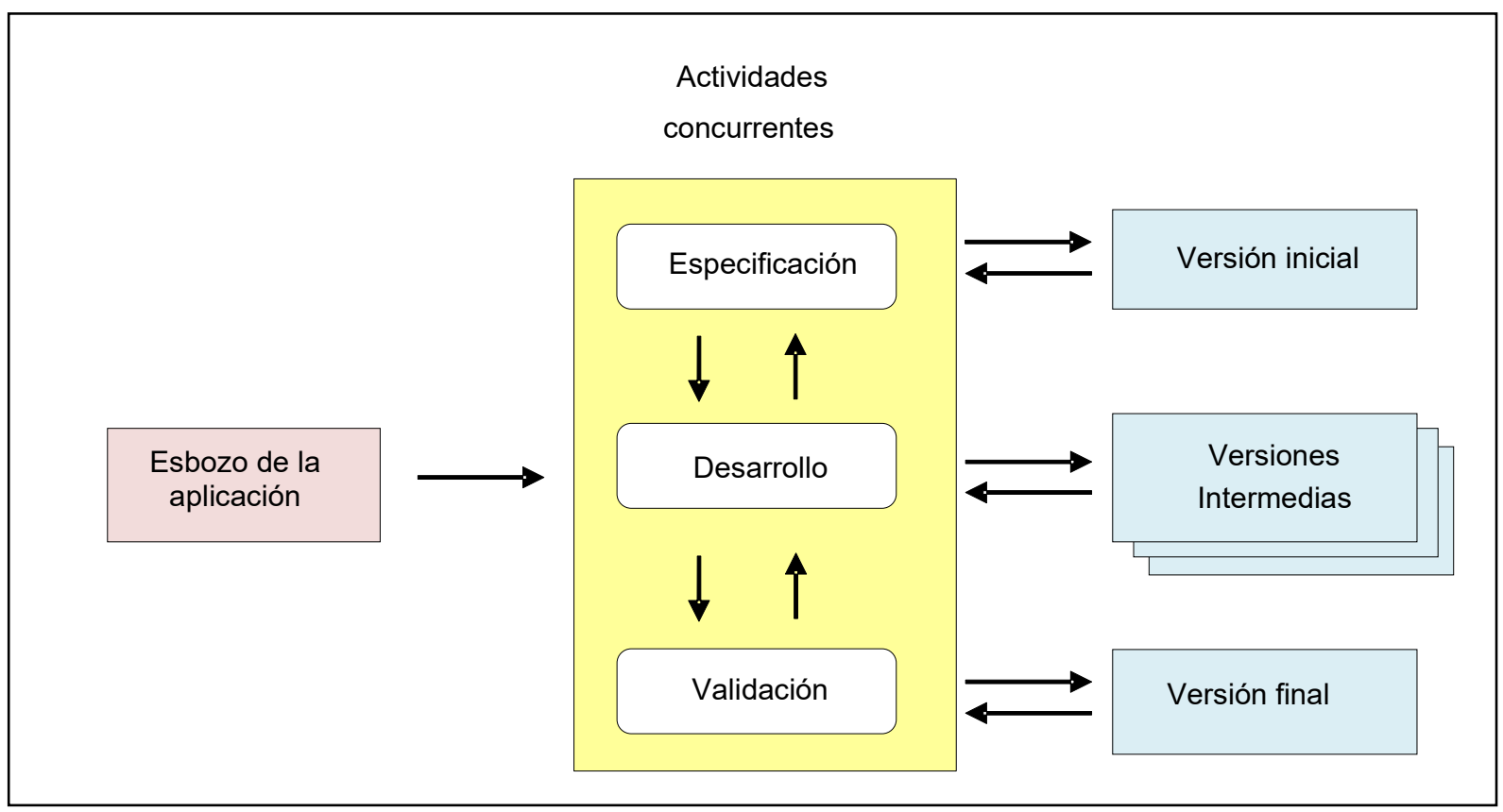

Fig. 1. Esquema del desarrollo incremental, adaptado de Sommerville (2011) 
El número de aplicaciones diseñadas para estudiantes con dificultades específicas de aprendizaje (DEA) se está multiplicando exponencialmente en la última década (Olakanmi et al., 2020). Muchos desarrolladores han diseñado aplicaciones móviles para ayudar a estos niños con dificultades específicas de la lectura, también conocida como dislexia, o con dificultades para la escritura (disgrafía y disortografía). Skiada et al. (2014) implementaron una aplicación compatible con dispositivos Android para fomentar en estos niños las habilidades de resolución de problemas matemáticos y de comprensión de la lectura y corrección ortográfica, apoyándose en entrenamientos de memoria a corto plazo, que es la memoria básica que influye en todo aprendizaje. Tariq y Latif (2016) diseñaron una aplicación móvil para tabletas que persigue mejorar el rendimiento del aprendizaje en la escritura de niños con dislexia. Demostraron que su uso incrementaba la atención, que se focalizaba más en las tareas de aprendizaje, en comparación con los soportes tradicionales en papel. Lindeblad et al. (2017) demostraron efectos positivos a largo plazo tanto en el rendimiento en las habilidades adquiridas mediante juegos de lectura y escritura implementados en tabletas como en la motivación y adherencia hacia la práctica de lectura.

Estos estudiantes no consiguen aprender al mismo ritmo que sus compañeros, sin que esto se pueda atribuir a deficiencias de tipo intelectual, sensorial, motor, de trastornos psiquiátricos o por falta de esfuerzos educativos (Asociación Estadounidense de Psiquiatría, 2014). Se atribuye estas DEA a disfunciones de tipo neurológico que acompañaran a las personas durante toda su vida, con diferente impacto, en función de muchas variables, como la personalidad, la gravedad del trastorno, los apoyos recibidos, etc. Algunas de ellas consiguen superar sus dificultades y alcanzan buen desempeño académico, mientras que otras fracasan en su escolarización (Goldberg et al., 2003). El problema siempre se manifiesta en los primeros cursos de la educación primaria y se agrava a medida que aumenta la exigencia en las habilidades básicas de lectura, escritura y cálculo. Son estudiantes que, inexplicablemente, no desarrollan las habilidades de lenguaje escrito (lectura y escritura) o las habilidades para el cálculo y razonamiento numérico al ritmo esperado. Como consecuencia, se produce un retraso general en el rendimiento escolar, porque esas habilidades funcionan como instrumentos para el resto de los aprendizajes académicos. También son muy frecuentes las reacciones emocionales adversas debido a las consecuencias sociales de esta falta de aprendizaje (Zuppardo et al., 2020).

Las deficiencias en el procesamiento fonológico parecen estar en la base de los problemas cognitivos que desencadenan las dificultades de aprendizaje del lenguaje escrito en las personas con dislexia (Snowling et al., 2020). El procesamiento fonológico tiene tres componentes que son deficitarios es todas estas personas: la capacidad de nombramiento rápido, la memoria fonológica y la conciencia fonológica. El nombramiento rápido se mide pidiendo a la persona que pronuncie lo más rápidamente posible el nombre de objetos que conoce, por ejemplo cinco figuras geométricas básicas. Supone la capacidad de acceder mentalmente muy rápido y de forma alternativa a etiquetas verbales almacenadas en la memoria a largo plazo. La memoria fonológica se mide pidiendo a la persona que repita secuencias de sílabas que no forman una palabra, pero que siguen las normas del idioma. Supone la capacidad de descodificar los sonidos, almacenarlos en la memoria a corto plazo por unos instantes y volver a refrescar la secuencia para pronunciarla. La conciencia fonológica es la capacidad de manipular mentalmente la estructura fónica de la palabra, identificando las sílabas y los sonidos aislados. Permite realizar operaciones mentales que nos hacen conscientes, por ejemplo, de que las palabras sol y suelo comienzan por el mismo sonido o que la diferencia entre las palabras nuevo y muevo reside únicamente en el primer sonido. Se mide con tareas que implican estas operaciones mentales como, por ejemplo, identificar rimas o aliteraciones.

Estas capacidades fonológicas permiten la automatización en muy poco tiempo de los procesos básicos de lectura y escritura: la relación bidireccional entre sonidos y letras y la capacidad para realizar combinaciones de letras para formar sílabas y de sílabas para formar palabras. Estas habilidades básicas, denominadas de descodificación, son automatizadas con eficacia por los niños en los primeros años de la educación básica. Son las habilidades que se conocen en la escuela como de mecánica lectora. Permiten a un niño saber cómo suena lo que pone en el texto y le conecta con las palabras almacenadas en su mente, dando paso a los procesos de comprensión. Los niños normotípicos alcanzan una pericia considerable durante los primeros tres cursos escolares. Una prueba de ello es que cuando leen en voz alta suenan prácticamente igual que cuando hablan. Sin embargo, las personas con dislexia tardan años en ser competentes para descodificar los textos y dedican gran parte de su energía cognitiva a identificar de forma tortuosa como debería sonar las combinaciones de letras y para reconocer las palabras (Cadavid-Ruiz et al., 2019). Los niños con dislexia cuando leen en voz alta suenan vacilantes, lentos y silabeantes, además de cometer muchos errores de transcripción. Los que leen en lenguas transparentes ortográficamente, como el español, holandés o rumano, alcanzan antes un nivel mínimo de exactitud que los que leen en lenguas opacas como el inglés o el francés. El idioma no influye en la velocidad a la que las personas realizan estos procesos de descodificación de la lectura. En ningún idioma las personas con dislexia alcanzan la velocidad de lectura de las personas sin dislexia (Defior y Serrano, 2012). Estas características hacen que están personas sean eternos aprendices de las habilidades básicas de lectura, mientras que sus compañeros se convierten en apenas tres cursos escolares en expertos intérpretes del código ortográfico. 
Los estudios parecen demostrar, en contra de lo que podría suponerse, que los niños con dislexia tienen la misma dificultad para almacenar representaciones ortográficas formadas por sílabas simples y frecuentes que por sílabas más complejas y menos frecuentes (Suárez-Coalla y Cuetos, 2017; Álvarez-Cañizo et al., 2018). La variable que parece ser más importante es el número de veces que el niño debe ser expuesto a la palabra para almacenar su forma ortográfica. Este número es siempre mucho mayor que el de niños que no padece dislexia. Es por esto por lo que los juegos informáticos pueden tener un papel importante para favorecer el aprendizaje de la escritura. El objetivo principal de este trabajo es el desarrollo de una aplicación compatible con un sistema operativo Android para niños con dificultades específicas de aprendizaje de la lectura y la escritura.

\section{MATERIALES Y MÉTODOS}

Se detallan los materiales y métodos empleados, en cuatro subsecciones: análisis de requerimientos, diseño y desarrollo de software, validación y clasificación del vocabulario.

\section{Análisis de requerimientos}

La aplicación debe ser compatible con dispositivos con un sistema operativo Android y proporcionar ejercicios que impliquen combinar sílabas para formar palabras. En la configuración del programa el educador debe poder seleccionar: 1) si la actividad implica escritura o solo lectura; y 2) el tipo de sílabas que forman las palabras que aparecerán en el ejercicio.

\section{Diseño y desarrollo de software}

En primer lugar, se analizaron las características del software especificadas por el equipo pedagógico. Posteriormente se realizó un estudio de aplicaciones con características similares y se optó por utilizar el mismo sistema de recompensa al acertar un ejercicio mediante la presentación de refuerzos auditivos y visuales (Máñez-Carvajal y Cervera-Mérida, 2021; Máñez-Carvajal y Máñez, 2020).

Para llevar a cabo el desarrollo informático se ha empleado Basic4android, un sencillo y potente entorno de desarrollo especialmente destinado a generar aplicaciones para dispositivos Android. El lenguaje que emplea es BASIC, similar a Visual Basic con soporte adicional para objetos. Además, proporciona numerosas bibliotecas que facilitan el desarrollo de aplicaciones avanzadas. Con respecto al diseño de la interfaz de usuario se empleó la herramienta Visual Designer, que permite gestionar los diferentes objetos de la interfaz. De esta manera, se ajustaron las posiciones y tamaños de los elementos empleados en la pantalla del dispositivo (Figura 2).

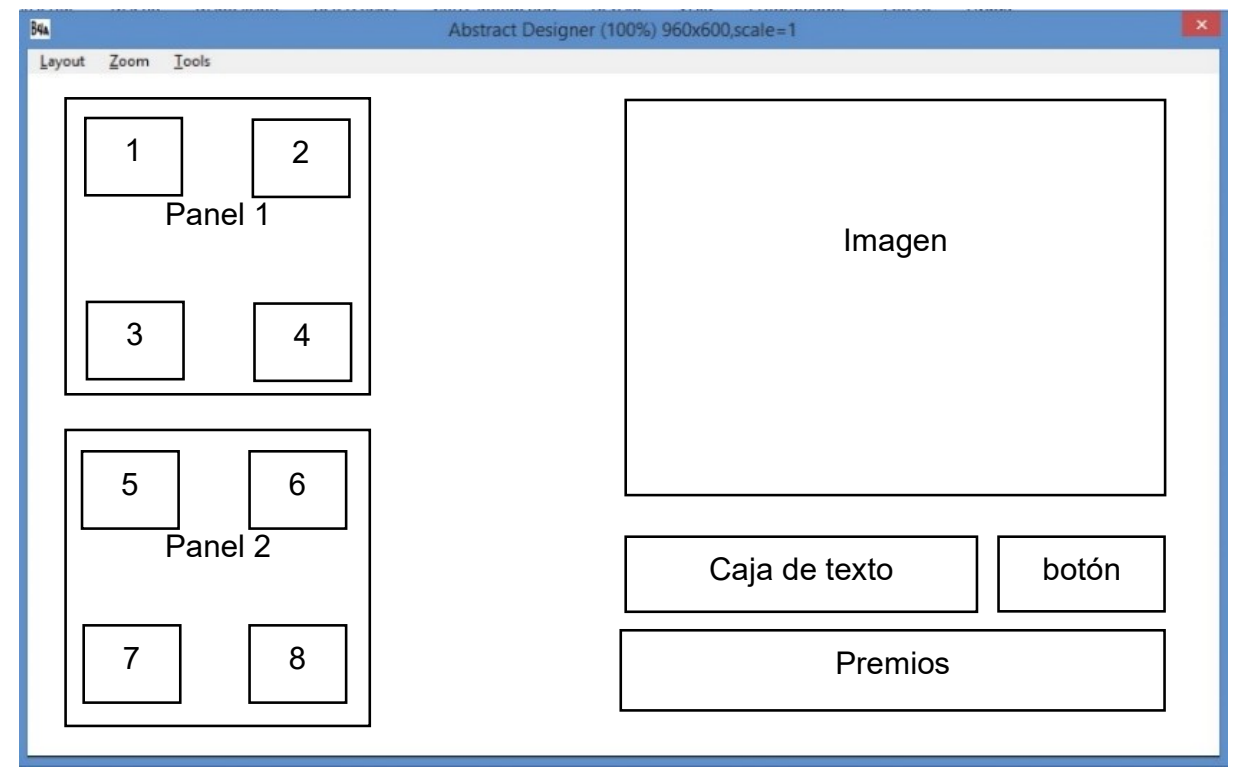

Fig. 2. Herramienta Visual designer

Para evitar la repetición de ejercicios en la misma posición, se utilizaron funciones que generan números aleatorios y vectores auxiliares. Las sílabas que forman cada palabra se representan en etiquetas de texto ubicándose en distintas posiciones en la pantalla. Con respecto a los recursos empleados, las imágenes, sonidos y ficheros de texto se almacenaron en el dispositivo, lo que permite al usuario trabajar con el programa sin la exigencia de estar conectado a Internet. Se consideró hacer uso de bases de datos, sin embargo, teniendo en cuenta el tipo de desarrollo y los recursos facilitados para el mismo, se optó por utilizar ficheros de texto. 


\section{Validación}

Se presentó al equipo pedagógico una primera versión de la aplicación que contaba únicamente con un tipo de sílabas, se modificaron aspectos de diseño, tamaño de las imágenes, fuente y disposición de los elementos en la interfaz de usuario. Tras la evaluación de una versión intermedia, se implementó la versión final del programa con los cinco conjuntos de sílabas.

\section{Clasificación del vocabulario}

El vocabulario se ha elegido entre palabras de alta frecuencia de uso para las edades entre 6 y 10 años según el Vocabulario Básico de EGB Espasa Calpe-MEC. Las sílabas se han clasificado en fáciles y difíciles, atendiendo a criterios de relación biunívoca entre sonido y grafema, y también a criterios empíricos que indican que algunas letras son más fáciles de aprender por la mayoría de los alumnos. Consta de palabras clasificadas como fáciles, con estructuras silábicas sencillas compuestas únicamente por vocal o consonante-vocal (35 bisílabas, como 'ala' y 23 trisílabas como 'camisa') y palabras difíciles (79 bisílabas, 62 trisílabas y 25 tetrasílabas) con estructuras silábicas complejas como consonante-consonante-vocal (alfombra). Las imágenes se han elegido tratando de que su asociación con la palabra fuera obvia. La tabla 1 muestra una representación del vocabulario que emplea la aplicación.

Tabla 1 Vocabulario de la aplicación

\begin{tabular}{|c|c|c|c|c|}
\hline Bisílabas fáciles & Bisílabas difíciles & Trisílabas fáciles & Trisílabas difíciles & Tetrasílabas \\
\hline ajo & agua & abeja & abrigo & aceitunas \\
\hline ala & algas & abeto & aceite & acordeón \\
\hline boca & ángel & abuela & acelga & alcachofa \\
\hline bola & árbol & abuelo & águila & almohada \\
\hline boli & arroz & anillo & aguja & amapola \\
\hline bota & asno & antena & ajedrez & ambulancia \\
\hline café & avión & asiento & alambre & avioneta \\
\hline caja & balcón & caballo & albañil & batidora \\
\hline cama & balón & cadena & alfiler & bicicleta \\
\hline
\end{tabular}

\section{RESULTADOS Y DISCUSIÓN}

El uso de la aplicación es totalmente intuitivo y no exige ninguna preparación. El educador debe decidir en la pantalla de configuración del ejercicio: 1) la cantidad y dificultad de sílabas que forman las palabras que aparecerán en el ejercicio y 2) si la actividad implica escritura o solo lectura. Las opciones del programa permiten elegir el nivel de complejidad lingüística al que se expone al niño en cada ejercicio y la tarea que deberá realizar. Esta toma de decisiones se ha simplificado al máximo para que los educadores perciban el manejo como intuitivo. La primera decisión que afrontan es si el ejercicio es fácil o difícil y si contiene bisílabas, trisílabas o tetrasílabas. La segunda decisión es si el ejercicio solamente implica una tarea de lectura o si implica lectura y escritura. La figura 3 muestra una captura de pantalla donde el educador toma estas decisiones.

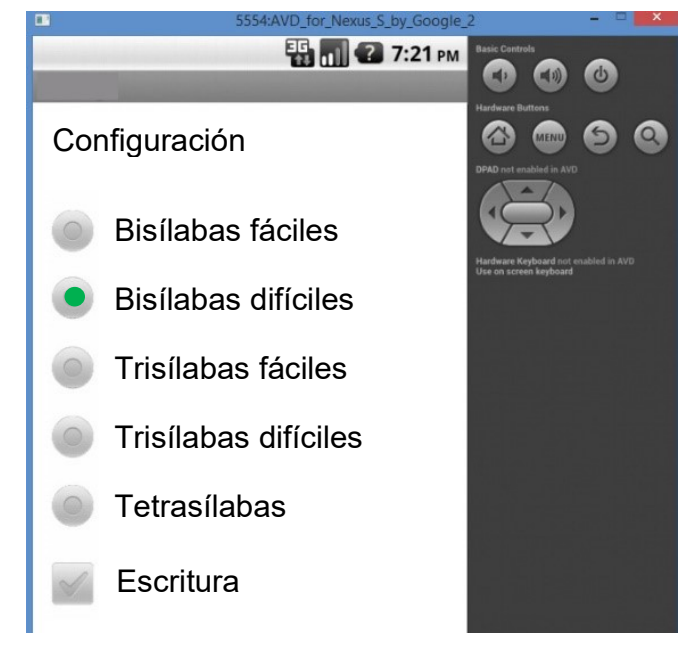

Fig. 3. Configuración del programa 
Las primeras veces que el niño utiliza la aplicación debería estar asesorado por un educador que moldeara el procedimiento para que evitar los tanteos irreflexivos. El educador debe explicar la tarea y realizar en voz alta, explicando cada paso y reflexionando sobre las opciones. Los niños que no tengan totalmente automatizada la lectura de sílabas aisladas y duden de cómo se pronuncian, deberían recibir ayuda del educador pues el programa no trabaja este conocimiento, sino que lo concibe como un requisito para entrenar una habilidad superior, la del acceso al léxico.

Una vez el educador ha tomado las decisiones anteriores, el niño verá en pantalla una fotografía y dos conjuntos de sílabas desordenadas espacialmente y aleatorizadas en cada presentación. La fotografía activará en la mente del niño una palabra que ha sido elegida entre las frecuentes para el rango de edades a los que se destina el programa. Funciona como estímulo facilitador y anticipador del procesamiento lingüístico, que hará más sencillo el proceso de descodificación de las sílabas. Por ejemplo, cuando el niño ve la foto de un pájaro activa en su mente la huella acústica de la palabra o representación fonológica (pa+ja+ro). A continuación, tiene que tomar la decisión si las sílabas que ve son compatibles con esa huella o no. El proceso de descodificación de las sílabas, es decir, de conversión de letra a sonido, tiene una finalidad, la de buscar la compatibilidad con los sonidos que forman la palabra. De esta manera, se espera que se fortalezca la unión entre la representación fonológica de la palabra o huella acústica y su representación o huella ortográfica. Cada ejercicio repite esta operación con diez palabras diferentes seleccionadas al azar entre las que cumplen las condiciones. Si el ejercicio solo exige la lectura, la tarea del niño consiste en decidir cuál es el conjunto de sílabas que forma la palabra a la que se refiere la imagen, pulsando en el recuadro que contiene el conjunto de sílabas adecuado y recibiendo en ese momento la retroalimentación positiva o negativa (Figura 4).

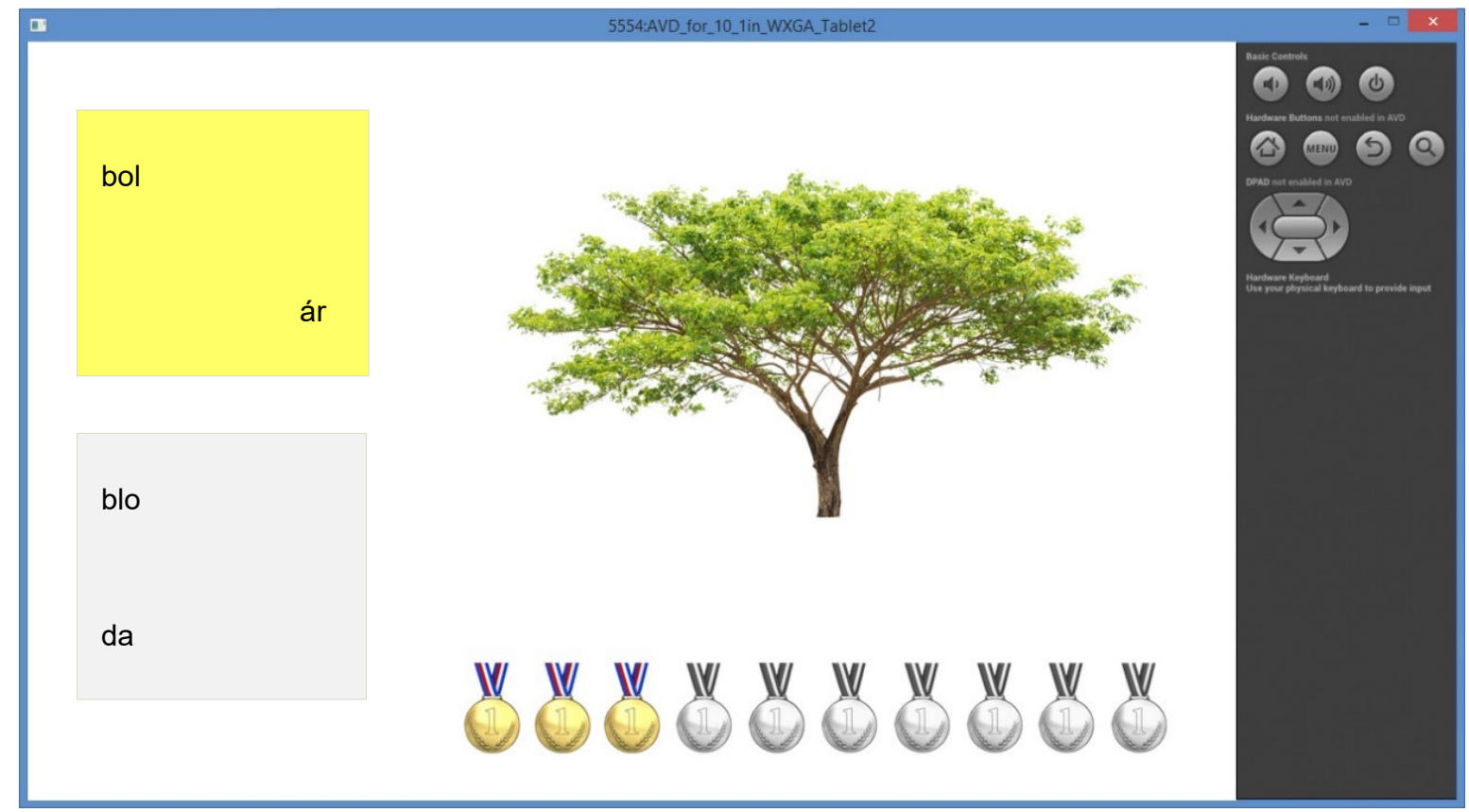

Fig. 4. Ejercicio de lectura de palabras

La respuesta del niño implica la identificación de los sonidos de cada sílaba, pero el procesamiento se ve favorecido enormemente por el fenómeno de anticipación mental activado por la fotografía. La tarea no es descodificar unas sílabas para acceder a una palabra que el niño ignora, sino: descodificar las sílabas de la palabra que ya tiene en la mente. Este fenómeno de anticipación facilita y da sentido al proceso de descodificación, que está seriamente afectado en los niños con dislexia. Si el niño elige las sílabas incorrectas recibe un refuerzo auditivo negativo y el conjunto de sílabas queda atenuado. El programa no avanza hasta que el niño corrige y pulsa las sílabas correctas, que quedan resaltadas, y entonces recibe refuerzo positivo tanto visual como sonoro. Los reforzadores sonoros indican acierto o fallo al seleccionar el conjunto de sílabas correspondiente. Al pulsar la respuesta correcta el programa activa una de las diez medallas indicando el acierto. El niño sabe en todo momento cuántos ítems ya ha resuelto y cuántos le quedan por resolver. Esto es un sistema de refuerzo muy potente para los niños a los que la tarea les resulta difíciles

En el caso de que el ejercicio implique la lectura y escritura el proceso es el mismo que hemos descrito, pero una vez el niño ha seleccionado las sílabas adecuadas y recibida la retroalimentación positiva aparece en pantalla un recuadro de texto en el que debe escribir la palabra (Figura 5). Se trata de una tarea de copia, dado que el conjunto de sílabas sigue estando en pantalla. Como las sílabas están desordenadas espacialmente y aleatorizadas en cada presentación, la tarea exige una dosis de atención considerable y 
habilidades de conciencia fonológica para refrescar en la memoria la secuencia fónica y transcribir mediante el teclado la secuencia ortográfica. Este es el núcleo del procesamiento cognitivo donde la aplicación pretende incidir. La fotografía permite refrescar constantemente la palabra, descargando la memoria de este proceso. El niño ha seleccionado previamente la secuencia de sílabas en el paso anterior, que implica una tarea de lectura o descodificación de las sílabas. El último paso consiste en enfrentarse a una tarea ortográfica que implica la escritura de la secuencia de letras, teniendo como facilitador el modelo desordenado de sílabas.

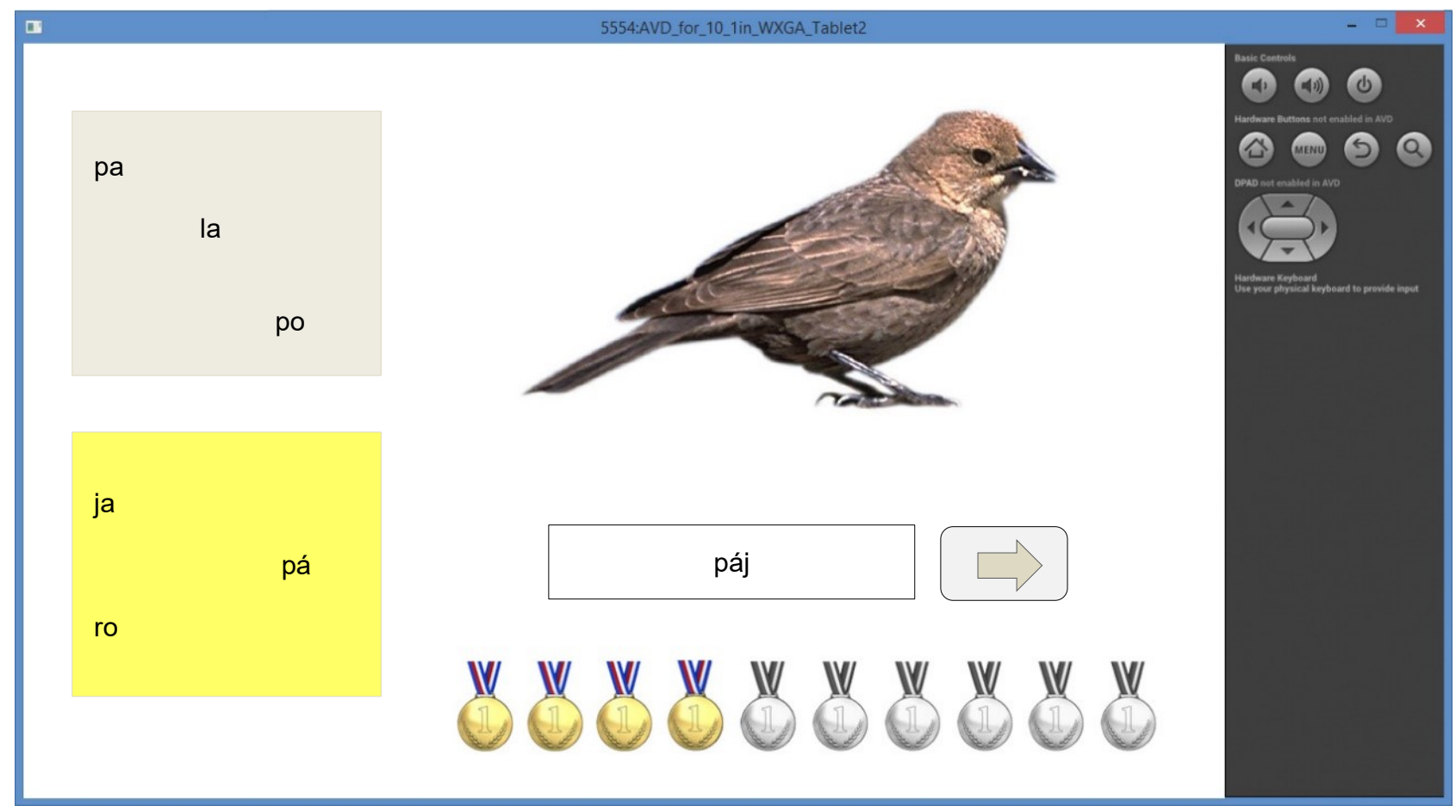

Fig. 5. Ejercicio de escritura de palabras

En un metanálisis reciente se ha comprobado el efecto relativamente positivo de las tecnologías de ayuda para los niños con dificultades de aprendizaje de la lectura y escritura. Al parecer los efectos positivos se obtienen más usando las tecnologías basadas en el procesamiento de textos, los multimedia y los hipertextos y no tanto en los conversores voz a texto y bolígrafos inteligentes, que tienen resultados positivos con algunas personas e indiferentes con otras (Perelmutter et al., 2017). El efecto positivo general de usar juegos como estrategia de aprendizaje en las aplicaciones destinadas a niños con DEA parece estar demostrado (Lämsä et al., 2018).

La aplicación diseñada se basa en este efecto. La aplicación contiene ejercicios que implican combinar sílabas para formar palabras. Pretende reforzar la conciencia fonológica y facilitar el procesamiento lingüístico y de memoria. El entrenamiento repetido en los ejercicios de conversión entre sonidos y grafemas es necesario para que estos niños almacenen representaciones mentales ortográficas precisas y estables que mejoren sus habilidades de lectura y escritura. Los niños sin dificultades específicas de aprendizaje generan estas representaciones mentales de la ortografía de las palabras de una forma rápida tras pocos intentos de lectura. Sin embargo, los niños con dificultades específicas de aprendizaje necesitan muchas más repeticiones (Suárez-Coalla y Cuetos, 2017). La aplicación consigue hacer más tolerable las repeticiones necesarias para generar aprendizaje al proporcionar una dosis extra de motivación.

\section{CONCLUSIONES}

De acuerdo a los resultados de este estudio se pueden extraer las siguientes conclusiones principales: 1) Se ha diseñado la aplicación para facilitar el aprendizaje de lectura y escritura de palabras aisladas a niños con DEA; 2) Se pretende incidir en los procesos básicos de identificación de las sílabas que componen las palabras y asociación con los grafemas correspondientes; 3 ) En la tarea de lectura se proporcionan dos ayudas para facilitar el procesamiento cognitivo: la anticipación mental de la palabra, que activa su representación acústica, y la visión de las sílabas correspondientes frente a sílabas distractoras; 4) En la tarea de escritura se permite la copia, pero se exige una reconstrucción de la secuencia, lo que implica reforzar las habilidades de conciencia fonológica; y 5) El software desarrollado es compatible en tabletas con sistemas operativos Android y posibilita acceder a los contenidos en cualquier momento y lugar, sin la necesidad de Internet, permitiendo a los estudiantes una mayor flexibilidad para trabajar a su propio ritmo. 


\section{REFERENCIAS}

Alarcón-Aldana, A. C., Urrutia-Pinilla, J., y Callejas-Cuervo, M., Aplicación Móvil para la Administración de Variables Físicas en Ciclismo al Aire Libre, http://doi.org/10.4067/S0718-07642016000400019, Inf. Tecnol., 27(4), 175-182 (2016)

Álvarez-Cañizo, M., Suárez-Coalla, P., y Cuetos, F., The role of sublexical variables in reading fluency development among Spanish children, https://doi.org/10.1017/S0305000917000514, J. of Child Language, 45(4), 858-877 (2018)

Cáceres, L., y Ossandón, A., Desarrollo de una aplicación móvil para reconocimiento de personas después de una catástrofe natural utilizando la tecnología NFC, http://doi.org/10.4067/S0718-33052018000500028, Ingeniare Revista Chilena de Ingeniería, 26, 28-44 (2018)

Cadavid-Ruiz, N., Jiménez-Jiménez, S., Quijano-Martínez, M.C., y Solovieva, Y., Corrección de las dificultades psicopedagógicas de la lectura en español, http://dx.doi.org/10.12804/revistas.urosario.edu.co/apl/a.6628, Avances en Psicología Latinoamericana, 37(2), 361-374 (2019)

Choque-Tolmo, B. M., Villalobos-Abarca, M. A., y Herrera-Acuña, R. A., Desarrollo de un software web para la gestión de planes de negocios, http://doi.org/10.4067/S0718-07642020000400045, Inf. Tecnol., 31(4), 45-60 (2020)

Defior, S., y Serrano, F., Dislexia en español: bases para su tratamiento y diagnóstico, Dislexia: Definición e intervención en hispanohablantes, $2^{\mathrm{a}}$ ed., El Manual Moderno S.A. de C.V., 15-36, Ciudad de México, México D.F. (2012)

Kraut, R., Policy Guidelines for Mobile Learning, $1^{\text {a }}$ ed, United Nations Educational, Scientific and Cultural Organization, París, Francia (2013)

Gironés, J. T., El gran libro de Android, $7^{\mathrm{a}}$ ed., Marcombo S.A., Barcelona, España (2018)

Goldberg, R. J., Higgins, E. L., Raskind, M. H., y Herman, K. L., Predictors of Success in Individuals with Learning Disabilities: A Qualitative Analysis of a 20-Year Longitudinal Study, https://doi.org/10.1111/1540-5826.00077, Learning Disabilities Research \& Practice, 18(4), 222 (2003)

Lämsä, J., Hämäläinen, R., y otros 3 autores, Games for enhancing basic reading and math skills: A systematic review of educational game design in supporting learning by people with learning disabilities, https://doi.org/10.1111/bjet.12639, British Journal of Educational Technology, 49(4), 596-607 (2018)

Lindeblad, E., Nilsson, S., Gustafson, S., y Svensson, I., Assistive technology as reading interventions for children with reading impairments with a one-year follow-up, https://doi.org/10.1080/17483107.2016.1253116, Disability and Rehabilitation: Assistive Technology, 12(7), 713-724 (2017)

Máñez-Carvajal, C., y Cervera-Mérida, J. F., Aplicación móvil para la automatización del proceso de reconocimiento de palabras, Información Tecnológica, 32(5) en prensa (2021)

Máñez-Carvajal, C., y Máñez, C., Adquisición de vocabulario básico I, Aquari, ISSN: 2695 9216, 2(1), 1-3 (2020)

Olakanmi, O. A., Akcayir, G., Ishola, O. M., y Demmans Epp, C., Using Technology in Special Education: Current Practices and Trends, Educational Technology Research and Development, 68(4), 1711-1738 (2020)

Papadakis, S., Kalogiannakis, M., Orfanakis, V., y Zaranis, N, The Appropriateness of Scratch and App Inventor as Educational Environments for Teaching Introductory Programming in Primary and Secondary Education, https://doi.org/10.4018/IJWLTT.2017100106, Int. J. Web-Based Learning \& Teaching Technologies, 12(4), 58-77 (2017)

Perelmutter, B., McGregor, K. K., y Gordon, K. R., Assistive technology interventions for adolescents and adults with learning disabilities: An evidence-based systematic review and meta-analysis,

https://doi.org/10.1016/j.compedu.2017.06.005, Computers \& Education,114,139-163 (2017)

Politi-Georgousi, S., y Drigas, A., Mobile Applications, An Emerging Powerful Tool for Dyslexia Screening and Intervention: A Systematic Literature Review, https://doi.org/10.3991/ijim.v14i18.15315, International Journal of Interactive Mobile Technologies (IJIM), 14(18), 4-17 (2020)

Pressman, R., Ingeniería del software: un enfoque práctico, $7^{\mathrm{a}}$ ed., Mc-Graw Hill interamericana, México D.F., México (2010)

Snowling, M. J., Hulme, C., y Nation, K., Defining and Understanding Dyslexia: Past, Present and Future, https://doi.org/10.1080/03054985.2020.1765756, Oxford Review of Education, 46(4), 501-513 (2020)

Sommerville, I., Ingeniería del software, $9^{a}$ ed., Pearson Educación S. A., Madrid, España (2011)

Skiada, R., Soroniati, E., Gardeli, A., y Zissis, D., EasyLexia: A Mobile Application for Children with Learning Difficulties, https://doi.org/10.1016/j.procs.2014.02.025, Procedia Computer Science, 27, 218-228 (2014)

Suárez-Coalla, P., y Cuetos, F., Formation of orthographic representations in Spanish dyslexic children: The role of syllable complexity and frequency, https://doi.org/10.1002/dys.1546, Dyslexia: An International Journal of Research and Practice, 23(1), 88-96 (2017)

Zuppardo, L., Fuentes, A. R., Pirrone, C., y Serrano, F., Las repercusiones de la Dislexia en la Autoestima, en el Comportamiento Socioemocional y en la Ansiedad en Escolares, https://doi.org/10.5093/psed2020a, Psicología Educativa, 26(2), 175-183 (2020) 